\title{
A novel approach for micro-antenna fabrication on metal substrate assisted by laser printing for communication systems in smart implants
}

caroline moura ( $\square$ caroline.materiais@gmail.com )

University of Minho: Universidade do Minho

Hugo Dinis

Universidade do Minho

Oscar Carvalho

Universidade do Minho

João Carneiro Neto

Universidade do Minho

\section{Paulo Mendes}

Universidade do Minho

\section{Rubens Nascimento}

Universidade do Minho

\section{Filipe Silva}

Universidade do Minho

\section{Research Article}

Keywords: Nd:YAG laser, smart implants, communication system, antenna, implants

Posted Date: April 6th, 2021

DOl: https://doi.org/10.21203/rs.3.rs-355649/v1

License: (c) (i) This work is licensed under a Creative Commons Attribution 4.0 International License. Read Full License 


\section{Abstract}

The market of orthopedic implants is one of the biggest in the medicine field and it is expected to grow significantly in the next years, mainly because of the continuously growing elderly population.

Despite the technological advances, the implant-adjacent biological issues contribute to the implants' failures, and consequently reduce its lifetime. The development of implants with self-healing capabilities has been explored as a reliable approach to tackle the aforementioned issues. This can be accomplished through the introduction of actuators, sensors and antennas, which ultimately falls into the concept of smart implants. Thus, this work presents an alternative approach for printing antennas in Ti6Al4V substrate by using laser technique, thus allowing for the integration of communication modules directly in the implant. The performance of the fabricated antenna was assessed through simulation and compared with an ideal condition. In both situations, the antenna presented the better results at high frequencies. Nevertheless, the defects arising from the experimental fabrication contributed in a negative manner the antenna's efficiency.

\section{Introduction}

Despite the technological advances in orthopaedic implants, the frequent occurrence of failures trigged by implant-adjacent biological issues, namely wear, loosening and misalignment, significantly reduce the implant's lifetime. At the same time, the orthopaedic implants market tends to grow in the years to come as the population ages [1]. In this framework, the demand for new long-lasting implants is imperative. The development of implants with self-healing capabilities has been explored as a reliable approach to tackle the aforementioned issues. This can be accomplished through the introduction of actuators and sensors devices, which ultimately falls into the concept of smart implants.

The integration of smart components, and consequently their proper functioning, is highly dependent on the communication system, which is comprised, among others, by an antenna, and local surface areas with the ability to sense and or actuate. The antenna is responsible to transmit a signal from the interior of the body to the exterior and vice versa, and hence make up the main communication component between the implant and the user.

In general, a combination of classical surface micromachining and self-assembly techniques, such as evaporation and sputtering, is used to obtain micro-antennas, mostly fabricated on insulating substrates [2-5]. However, the latter methods would involve a multi-step fabrication process if applied for manufacturing antennas on an implant's surface. In this regard, 3D printing is emerging as an enabling technology for a wide range of new applications, in which materials as sensors, actuators or antennas may be successively printed, leading to a component with smart function areas. The main advantage of $3 \mathrm{D}$ printing technology is the ability to fabricate components, with complex structures, in a single step process [6]. 
The following requirements must be met so that the antenna performs successfully when placed in the implant's surface, namely it must (i) be small and (ii) isolated from the metallic substrate. The most common approach to ensure the insulation of the antenna is to cover the substrate with a dielectric material. For this purpose, zirconia is the ideal candidate mainly due to its dielectrically properties. Its high permittivity and low loss-tangent values $\left(\varepsilon_{\mathrm{r}}=29 ; \tan \delta \approx 0\right)$ [7] allow the electrical currents to concentrate in the metal-based antenna path and prevent loss to the substrate, thus mitigating power loss [8].

With this in mind, this work presents an alternative approach to print a silver-based antenna in the titanium alloy substrate, as a component of the communication system, assisted by laser technology. Although this work will focus on the printing of the antenna, as a separate step, this method will further integrate a continuous processing design, from which the implant and all other components will be printed in a single step. The laser is applied as a versatile tool, whether for preparing the surface of the material in a subtractive way, by creating micro-cavities, or for printing the components, in an additively way. The final design of the implant with the printed communication system is schematically presented in Fig. 1.

\section{Experimental Details}

The objective of this work is the proposal of a fabrication method capable of yielding an antenna directly integrated in the metal substrate of an implant, during its fabrication, without significant modifications to its design and structure. For this, it is desired the design of a small antenna with little impact on the structure of the implant. Therefore, a wire antenna inside the substrate presents itself as a viable option that meets the aforementioned requirements. The design of such an antenna is presented in the next section of this work.

\subsection{Proposed antenna}

Bearing in mind that the preservation of the structural integrity of the substrate is of paramount importance, an antenna with a low impact on the substrate is proposed. Such antenna can be, for example, a loop antenna, and this antenna architecture will be considered in this work. To build such an antenna, it is necessary to deposit a conductive metal wire in the metal substrate and guarantee its electrical insulation from the substrate. This can be achieved by creating a small cavity in the substrate that is filled with an electrical insulator, i.e. a dielectric material, followed by the creation of a conducting wire in the insulator.

The antenna proposed in this work is presented in Figure 2. The dielectric material is zirconia, while the conducting metal of the antenna is silver.

\subsection{Simulation performance of the proposed antenna}


The model of the proposed loop antenna was constructed using Ansys HFSS, and the antenna was simulated to determine its potential performance. The antenna model was also inserted inside a box with the same dielectric properties of muscle tissue, in order to mimic the implantation environment of the device, in accordance to Figure 3.

The antenna's return loss and impedance are presented in Figure 4, while its radiation pattern at $2.6 \mathrm{GHz}$ and $8.4 \mathrm{GHz}$ are presented in Figure 5.

Firstly, the return loss also presents an unusual wavy pattern, which is also observed in its impedance, leading to the conclusion that such an effect occurs due to the characteristics of the structure being simulated, and not a simulation error such as a poorly placed radiation absorption boundary.

Analysing the return loss, one could infer that the antenna operates best at lower frequencies, as the return loss is lowest in this region, as opposite to higher frequencies, where a significant part of the signal that is injected into the antenna is immediately reflected to the source. Nevertheless, when comparing the radiation patterns at $2.6 \mathrm{GHz}$ and $8.4 \mathrm{GHz}$, it is possible to see that the antenna presents the highest gain at the higher frequency $(-44 \mathrm{~dB}$ versus $-26 \mathrm{~dB})$. These contradictory results lead to the conclusion that, despite the fact that the antenna accepts the most power at the lower frequencies, this power is not radiated, as it is lost to the substrate through the dielectric insulator. On the other hand, at higher frequencies the dielectric material becomes thicker, electrically speaking, and is capable of better insulating the antenna from the metal substrate. Consequently, even though it doesn't accept as much power at high frequencies, the power it accepts is radiated rather than lost to the substrate. This claim is supported by the analysis of the efficiency of the antenna at each of the studied frequencies, as it increases from $-44.7 \mathrm{~dB}$ at $2.6 \mathrm{GHz}$ to $-29.2 \mathrm{~dB}$ at $8.4 \mathrm{GHz}$, a $15 \mathrm{~dB}$ increase. Additional support for this conclusion comes from the analysis of the surface currents at the dielectric material, presented in Figure 6 . As it can be seen, the current that travels through the dielectric is higher at lower frequencies, thus validating the previous claim.

The next section of this paper presents a fabrication method that can be used to produce antennas such as the one designed here.

\subsection{Antenna Fabrication}

In this work, commercial Yttria-stabilized zirconia (3Y-TZP) powder with dispersion of $3 \mathrm{~mol} \%$ Yttria (Tosoh Corporation, Japan), with particle size of $40 \mathrm{~nm}$ (in agglomerated size of $60 \mu \mathrm{m}$ ) was used as the dielectric material and silver powder ( $\geq 99 \%$ pure) with an average grain size of $230 \mathrm{~nm}$, from Metalor Technologies-USA, was used as the conductor path of the antenna.

The proposed fabrication procedure consists of three steps, which are described below:

i. Deposition and laser sintering of silver powder

ii. Spray deposition and laser sintering of zirconia layer; 
iii. Micro-cavity creation in Ti6Al4V substrate by laser;

\section{i) Micro-cavity creation}

In this step, a micro-cavity is created on the surface of a circular Ti6Al4V titanium alloy target with $8 \mathrm{~mm}$ diameter and a thickness of $2 \mathrm{~mm}$ for subsequently receiving the insulating layer and then, the silver powder. For this purpose, a pulsed Nd:YAG laser (OEM Plus) with a wavelength of $1064 \mathrm{~nm}$ and $6 \mathrm{~W}$ of maximum power has been used. To create the micro-cavity, a strategy consisting of overlapping lines with different wobble amplitudes and scanning speeds was performed. Thus, a sequence of 10 wobbles with different widths and scanning speed was undertaken, aiming to create a rounded shape cavity through more even distribution of laser energy. In additional, each wobble amplitude was repeated in ascending sequence, in order to achieve greater depth, which resulted in 110 laser passages. Figure 7 shows a scheme of the laser strategy above mentioned.

\section{ii) Zirconia layer deposition}

In this step, 3Y-TZP powder was used as a dielectric material. After micro-cavity creation on titanium alloy surface, a zirconia layer was deposited on the surface by means of spray deposition process. Zirconia powder was dispersed in acetone and the suspension was sprayed using an air-brush on top of the titanium substrate. Before spraying, the solution was ultrasonically dispersed to avoid particle agglomeration. The sample was sprayed three times on the surface, in order to obtain a thick and dense layer. Then, the $\mathrm{Ti} 6 \mathrm{Al} 4 \mathrm{~V} / \mathrm{ZrO}_{2}$ layer was heated by using a $\mathrm{CO}_{2}$ laser (Bende $\mathrm{CO}_{2}$ laser marking) with an output power of $40 \mathrm{~W}$ and a spot size of $200 \mu \mathrm{m}$ for zirconia layer consolidation. The great advantage of this process is the possibility to sinter specific zones without compromising the substrate. Thus, a power of $20 \mathrm{~W}$ and a scanning speed of $200 \mathrm{~mm} / \mathrm{s}$ was applied on the surface, in a frequency of $20 \mathrm{~Hz}$. Figure 2b schematically illustrates the sintering process of zirconia layer.

iii) Silver powder deposition and laser sintering

To print the silver-based antenna, silver powder ( $\geq 99 \%$ pure) with an average grain size of $230 \mathrm{~nm}$, (Metalor Technologies-USA) was compacted into the cavity created in step (i) on Ti6Al4V substrate. A pressure of $8 \mathrm{MPa}$ was applied to ensure the total accommodation of the powder. Then, a Nd:YAG laser (Sisma - $1064 \mathrm{~nm}$ of wavelength) with a spot size of $0.2 \mathrm{~mm}$ and $100 \mathrm{~J}$ of maximum energy has been used to sinter the silver powder and thus, generate the conductor path of antenna. An energy of $10 \mathrm{~J}$ was used in the sintering process. In this last step, a support Figure $2 \mathrm{c}$ illustrates this process. Each aforementioned step is schematically illustrated in Figure 8.

The cross-section of the produced antenna was analysed using scanning electron microscopy (SEM Nova NanoSEM 200, FEl, Netherlands) equipped with an Energy Dispersive Spectrometer (EDS). EDS analysis was performed using an energy of $15 \mathrm{keV}$ by means of a probe with spot size of $5.5 \mathrm{~mm}$, corresponding to a current of $9.2 \mathrm{nA}$. 


\section{Results And Discussion}

\subsection{Antenna characterization}

A photography of the fabricated antenna is shown in Fig. 9 below. The SEM image of the fabricated antenna is presented in Fig. 10 with the detailed dimensions of the layers produced according to the method described in Sect. 2.

As it can be seen, the micro-cavity is completely filled by dielectric material (zirconia) and the antenna around the middle of zirconia layer. Through the laser strategy used, a cavity with a more rounded shape was obtained, due to the energy distribution. Generally, when laser beam is used to remove material from the surface through ablation mechanism, the quality of the part is reduced by melt accretions and thermal damage; however, this problem can be reduced with shorter pulse duration, although in this case, the ablation efficiency is reduced. Despite this, laser ablation in the nanosecond range still offer great advantages and a good compromise between process quality and efficiency, depending on the strategy used to machine the surface and the material response.

In the laser strategy used, an increase of laser energy is obtained by increasing the wobble amplitude, which is associated with the cavity width. Laser energy density (E) can be calculated through the equation showed in a previous study [9]. For instance, by varying the wobble from 25 to $250 \mu \mathrm{m}$, the laser energy density increases from $0.3 \mathrm{~J} / \mathrm{mm}^{2}$ to $30 \mathrm{~J} / \mathrm{mm}^{2}$, respectively. As a consequence of that, the energy is more concentrated when a larger area is machined, which avoids an acute shape of the cavity.

After the micro-cavity creation by laser ablation, as open space for antenna printing, a dielectric layer is required. For this, zirconia is the choice material, as previously mentioned. Thus, after the spray deposition of zirconia on the substrate, this layer was irradiated by means $\mathrm{CO}_{2}$ laser source. Spray method was efficient for depositing a thick and homogeneous layer of zirconia. Others works have reported the spray deposition method as a simple and cheap technique for incorporating a material into a surface $[10,11]$.

In comparison to laser sintering of metals, which is well established, laser sintering of ceramics encounters some difficulties. Their high melting points make difficult to melt and high energy input is required. Thus, although Nd:YAG lasers is one of the currently popular laser sources for processing ceramics. The continuous wave $\mathrm{CO}_{2}$ laser is preferable, since its large wavelength of $10.6 \mu \mathrm{m}$ can be absorbed directly by most of ceramics, including $\mathrm{ZrO}_{2}$, but its focusing diameter is much bigger than that of the Nd:YAG laser, which leads to a decrease of the power intensity [12]. Additionally, the $\mathrm{CO}_{2}$ laser is less suitable for laser sintering of ceramics through the solid or liquid state mechanisms. In our case, as can be seen in the SEM images, the layer of zirconia is quite large, and they are not completely consolidated. Some grains are still released, requiring more laser energy to join them. In these cases, zirconia powder was mechanically trapped but not sintered. Even that, good adhesion was obtained of zirconia powder into the cavity. 
After the aforementioned process, silver powder was filled into the laser generated micro-cavity. Through Fig. 3 it is possible to see the silver path formed after the laser sintering process. It is possible to observe that silver powder was melted by laser and filled all the existing space after the dielectric deposition. As a consequence of the irregular roughness of the zirconia layer, silver path assumed an irregular shape with approximately $150 \mu \mathrm{m}$ of depth. A successful laser sintering depends relies on fully or partially melting of the solid powder granules. Thus, the formed melt wets the un-melted powder granules, which facilitates a viscous flow and further the local consolidation. For this, it is crucial that the laser energy per volume and time is controlled carefully when converting from electromagnetic radiation to thermal heat.

Further improvements are necessary to control the final geometry of the antenna during its manufacturing as also its centralization, in order to preserve the same dielectric thickness around for enhancing the antenna performance. Despite this, all the processes applied in this proposed solution for a micro-antenna fabrication proved to be promising alternatives to the commonly applied methods, by using laser technique. Generally, these micro-devices are printed on an insulator substrate (i.e. polymeric) by using standard lithographic or chemical deposition techniques $[13,14]$.

EDS analysis shows no silver oxide formation in the antenna path (Z3), after laser sintering. In additional, in the substrate zone (Z1), EDS presents Ti and Al peaks which is in agreement with Ti6Al4V composition. Vanadium is not present, because its amount is too low to be detected by this technique.

\subsection{Performance assessment of the fabricated antenna by simulation}

As seen in the previous section, the fabrication of the proposed antenna is not as straightforward as one would hope, and errors associated with the process itself mean that the final results aren't exactly like the antenna that was designed in the previous section. Consequently, the simulation model was updated to represent the measurements presented in Fig. 10. The detail of the uncentered silver line is presented in Fig. 11. The return loss and radiation patterns at $2.6 \mathrm{GHz}$ and $8.4 \mathrm{GHz}$ are also presented in Fig. 12.

As it can be seen from the results of Fig. 12, the return loss of the antenna is much higher in the entirety of the studied frequency range. This, in turn, is reflected in the decrease of the gain of the antennas at both the 2.6 and $8.4 \mathrm{GHz}$ scenarios. Nevertheless, the behaviour that was observed in the previous chapter, i.e. better results at higher frequencies, is still observed in this updated model, as expected, since the same justifications are still valid. The deterioration of the results can, in turn, be due to the smaller dielectric insulator thickness that was experimentally achieved, especially in the one side where it is as thin as $30 \mu \mathrm{m}$, thus leading to more significant losses through the dielectric to the substrate.

\section{Conclusions}

The antenna plays a key role in establishing the communication between the implant and the user, through signal and data transmission. The ability to incorporate the antenna on the implant's surface in a single step is of paramount importance to avoid further methods and to preserve its properties. The 
proposed approach has showed to be promising for printing antennas on Ti6Al4V-based implant surface. This achievement is made possible by using laser technique for creating the micro-cavity and also for consolidating the dielectric layer and the conductor path of the antenna.

The simulation results of the real case showed that the return loss of the antenna is much higher in the entirety of the studied frequency range. For both ideal and real cases, the better results are observed at higher frequencies and the deterioration of the outcomes in the real case can be due to the smaller dielectric insulator thickness that was experimentally achieved. Therefore, further simulations and experiments are needed in order to optimize the antenna efficiency by exploring higher and more accuracy dielectric thickness and its sintering conditions.

\section{Declarations}

\section{Author contribution:}

C.G. Moura: Investigation, writing; H. Dinis: Investigation, writing; O. Carvalho: Technical guidance; J.P. Carneiro Neto: Technical support; P.M. Mendes: Investigation, reviewing; R.M. Nascimento: Investigation, reviewing; F.S. Silva: Investigation, reviewing.

\section{Funding:}

This work has been supported by the FCT (Fundação para a Ciência e Tecnologia -Portugal) in the scope of the projects UID/EEA/04436/2019; Add.Additive_Manufacturing to Portuguese Industry_POCI-01-0247FEDER-024533, grant SFRH/BD/116554/2016, the CNPq (Conselho Nacional de Desenvolvimento Científico e Technológico) for the grant 205791/2014-0 and CAPES (Coordenação de Aperfeiçoamento de Pessoal de Nível Superior).

\section{Data availability:}

The datasets generated during and/or analyzed during the current study are available from the corresponding author on reason- able request.

\section{Conflict of interest}

The authors declare no competing interests

\section{Ethical approval}

Not applicable.

\section{Consent to participate}

Not applicable. 
Not applicable.

\section{References}

1. O'Connor C, Kiourti A (2017) Wireless Sensors for Smart Orthopedic Implants. J Bio- Tribo-Corrosion 3:1-8. https://doi.org/10.1007/s40735-017-0078-z

2. Pizzi M, De Martiis O, Grasso V (2004) Fabrication of self assembled micro reservoirs for controlled drug release. Biomed Microdevices 6:155-158.

https://doi.org/10.1023/B:BMMD.0000031753.02913.e2

3. Villani G, Bose R, Gabrielli A (2011) Evaluation of on-chip micro antennas for in vivo dosimetry application. Radiat Meas 46:1963-1966. https://doi.org/10.1016/j.radmeas.2011.06.022

4. Dhillon GS, Kaur S, Brar SK (2013) Perspective of apple processing wastes as low-cost substrates for bioproduction of high value products: A review. Renew Sustain Energy Rev 27:789-805. https://doi.org/10.1016/j.rser.2013.06.046

5. Singh I, Tripathi VS (2011) Micro strip Patch Antenna and its Applications: a Survey. Int J Comp Tech Appl 2:1595-1599

6. Lee JY, Tan WS, An J, et al (2016) The potential to enhance membrane module design with 3D printing technology. J Memb Sci 499:480-490. https://doi.org/10.1016/j.memsci.2015.11.008

7. Skrivervik AK, Merli F (2011) Design Strategies for Implantable Antennas. In: 2011 Loughborough Antennas \& Propagation Conference. pp 1-5

8. Asimina Kiourti and Konstantina S. Nikita (2012) A Review of Implantable Patch Antennas for Biomedical Telemetry: Challenges and Solutions. IEEE Antennas Propag Mag 54:210-228

9. Moura CG, Carvalho O, Gonçalves LM V, et al (2019) Laser surface texturing of Ti-6Al-4V by nanosecond laser: Surface characterization, Ti-oxide layer analysis and its electrical insulation performance. Mater Sci Eng C 104:109901.

https://doi.org/https://doi.org/10.1016/j.msec.2019.109901

10. Fujihara K, Kumar A, Jose R, et al (2007) Spray deposition of electrospun TiO2 nanorods for dyesensitized solar cell. Nanotechnology 18:2-7. https://doi.org/10.1088/0957-4484/18/36/365709

11. Ranga Rao A, Dutta V (2007) Low-temperature synthesis of TiO2 nanoparticles and preparation of TiO2 thin films by spray deposition. Sol Energy Mater Sol Cells 91:1075-1080.

https://doi.org/10.1016/j.solmat.2007.03.001

12. Regenfuss P, Streek A, Hartwig L, et al (2006) Principles of laser micro sintering. 17th Solid Free Fabr Symp SFF 2006 4:740-753. https://doi.org/10.1108/13552540710776151

13. V. Kyovtorov, I. Georgiev, S. Margenov, D. Stoychev, F. Oliveri DT (2017) New antenna design approach-3D polymer printing and metallization. experimental test at $14-18 \mathrm{GHz}$. Int J Electron Commun 73:119-128

14. Anacleto P, Mendes PM, Gultepe E, Gracias DH (2013) Micro antennas for implantable medical devices. 3rd Port Bioeng Meet ENBENG 2013 - B Proc 1-4. 


\section{Figures}

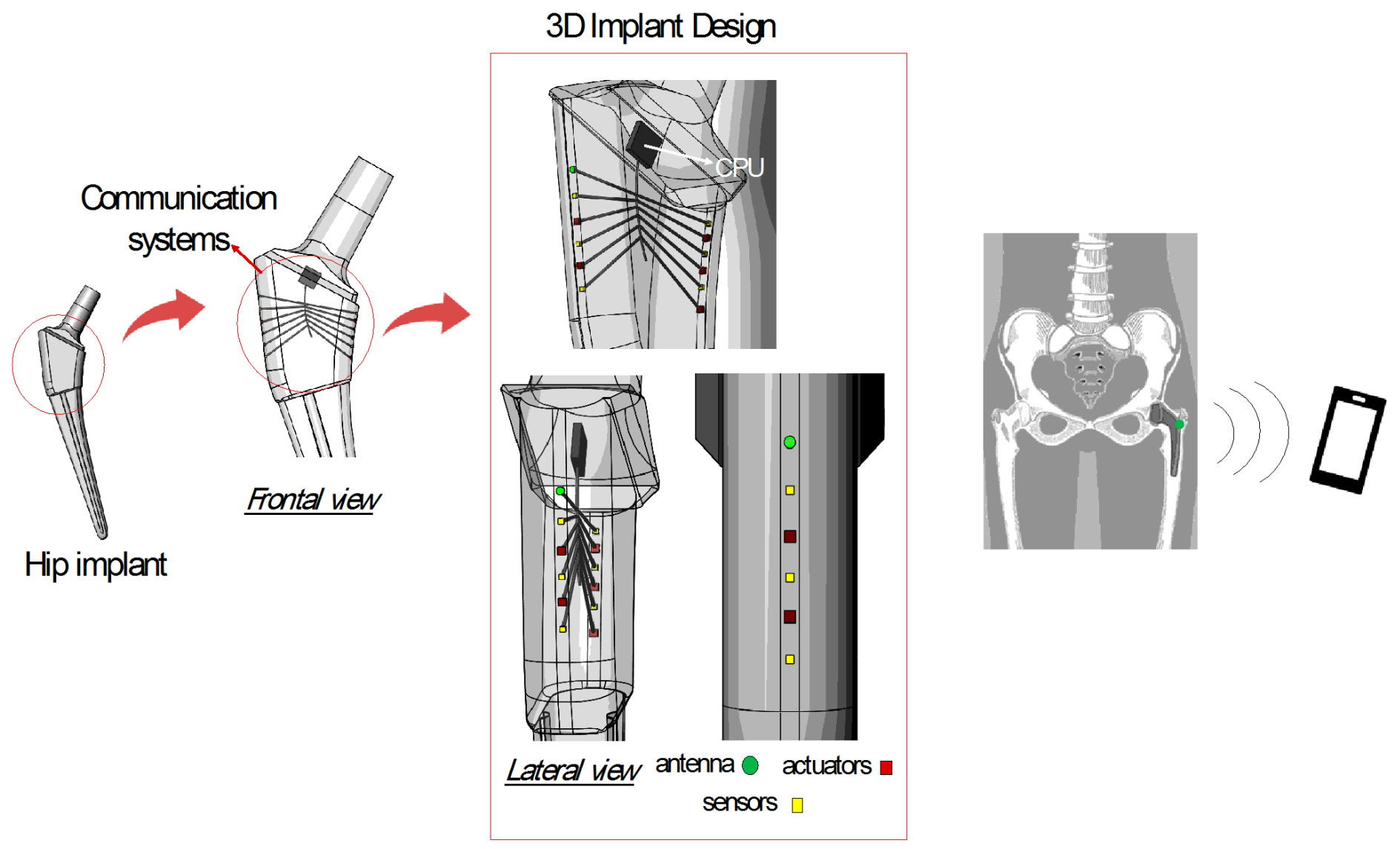

Figure 1

Schematically illustration of the final solution of Ti6Al4V-based implant with printed the communication system.

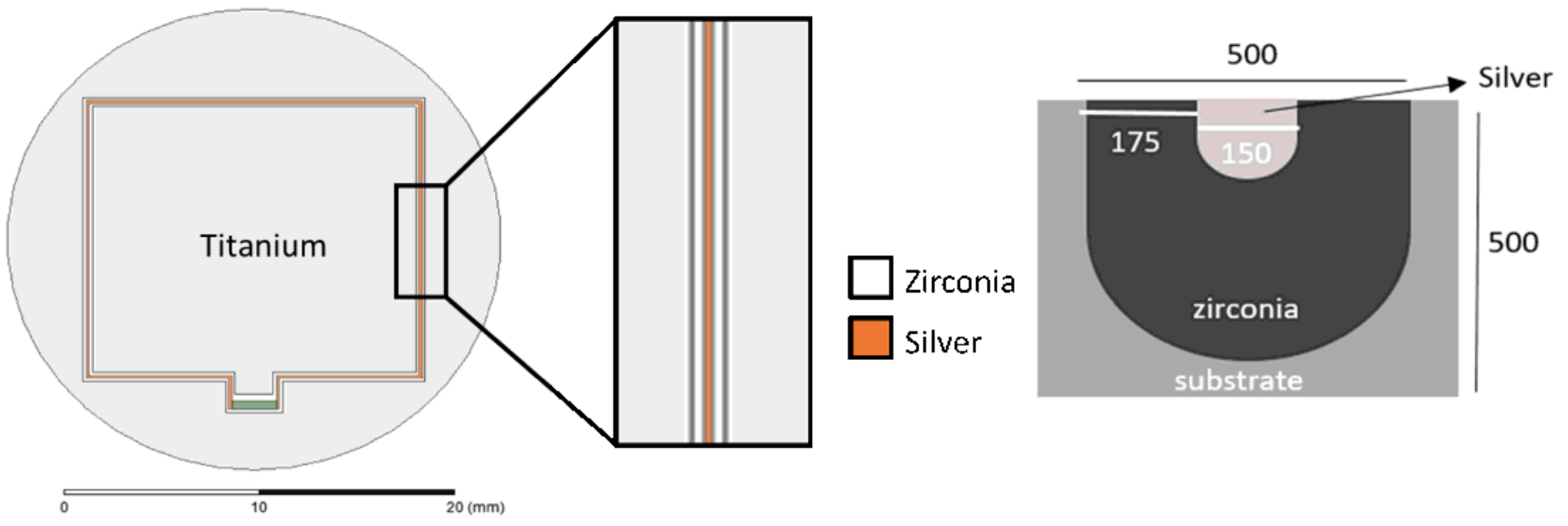

Figure 2 
Model of the proposed antenna, where the substrate is made of titanium, the dielectric material is zirconia and the metal path is made of silver (all units in $\mu \mathrm{m}$ ).

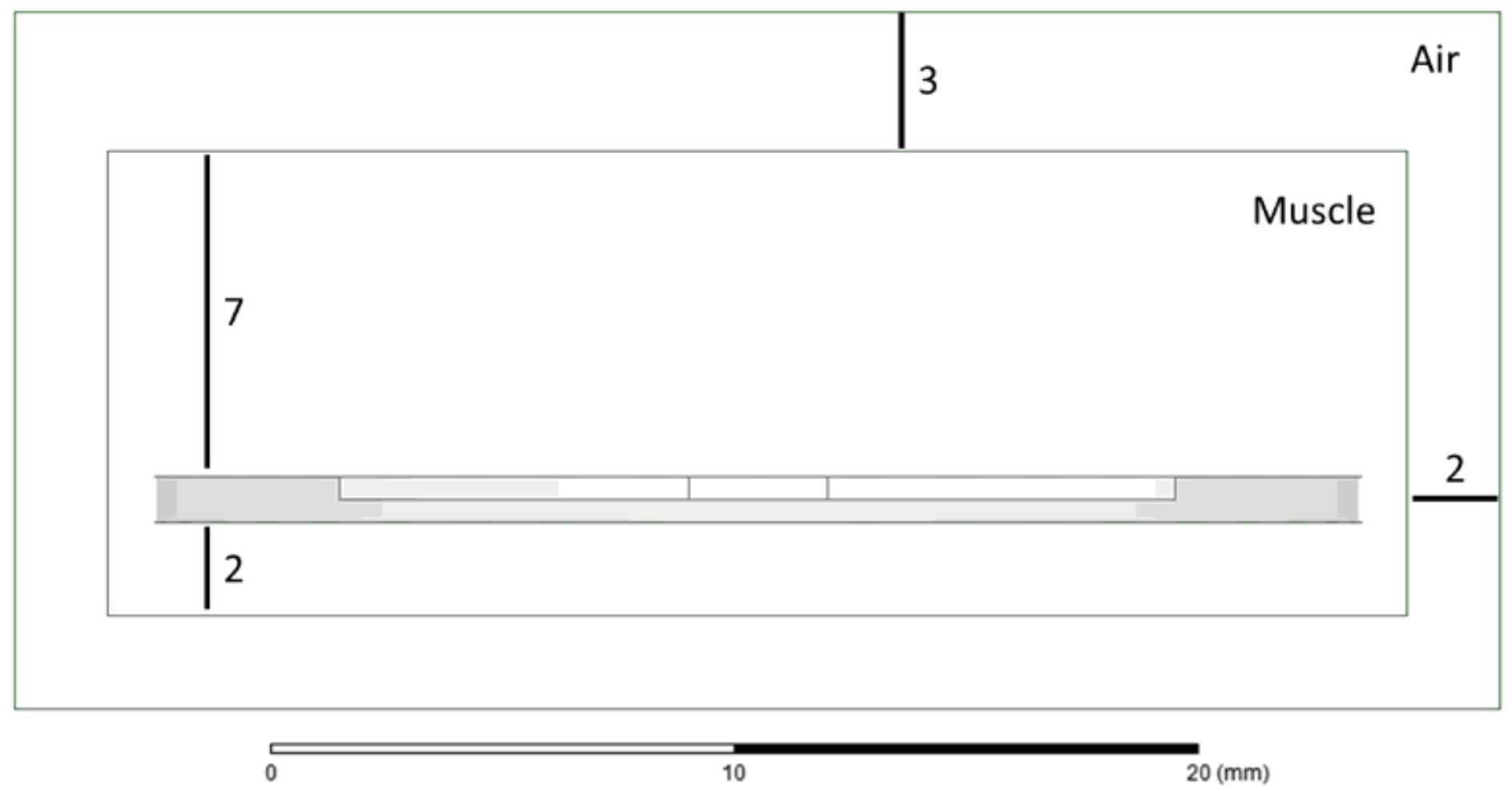

Figure 3

Muscle phantom placed around the model of the antenna.
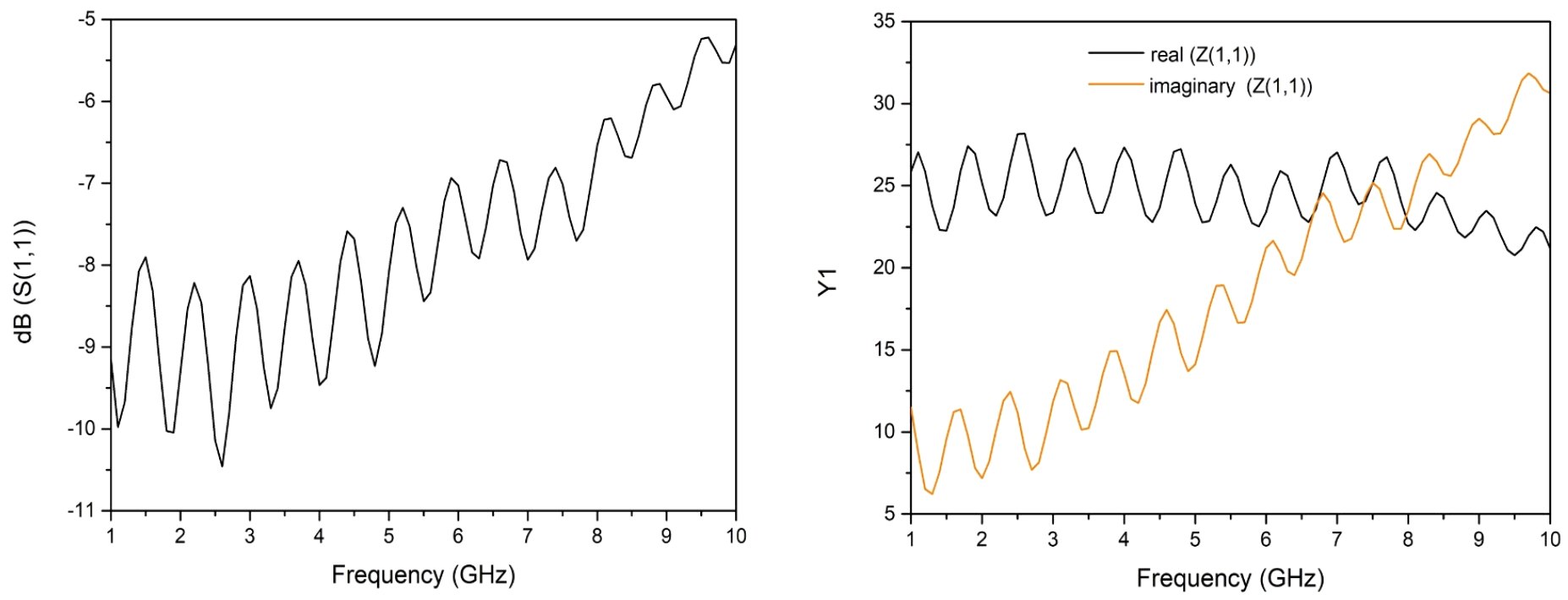

\section{Figure 4}

Return loss (top) and impedance (bottom) of the proposed antenna. 


\begin{tabular}{|c|}
\hline$d B$ (Rea 1izedGainTota1) \\
$-4.3821 E+01$ \\
$-4.5112 E+01$ \\
$-4.6403 E+01$ \\
$-4.7694 E+01$ \\
$-4.8985 E+01$ \\
$-5.0276 E+01$ \\
$-5.1567 E+01$ \\
$-5.2858 E+01$ \\
$-5.4149 E+01$ \\
$-5.5440 E+01$ \\
$-5.6731 E+01$ \\
$-5.8022 E+01$ \\
$-5.9313 E+01$ \\
$-6.0604 E+01$ \\
$-6.1895 E+01$ \\
$-6.3186 E+01$ \\
\hline
\end{tabular}
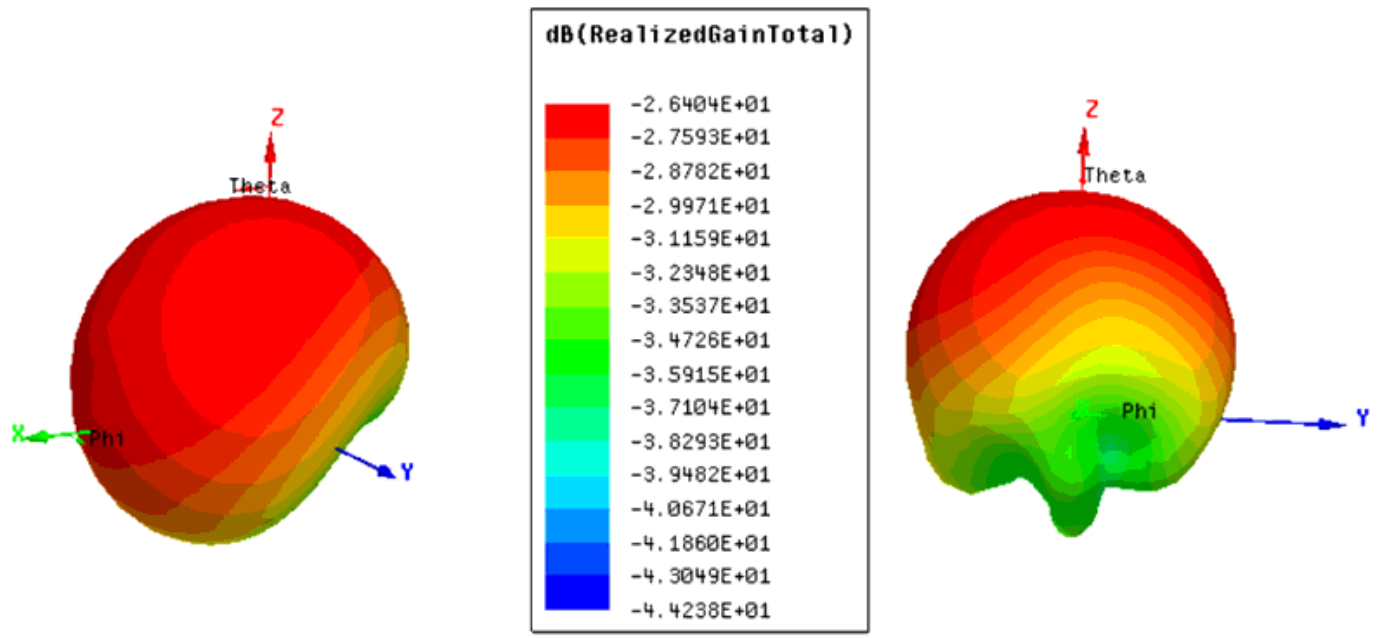

\section{Figure 5}

Radiation pattern of the antenna at $2.6 \mathrm{GHz}$ (left) and $8.4 \mathrm{GHz}$ (right).

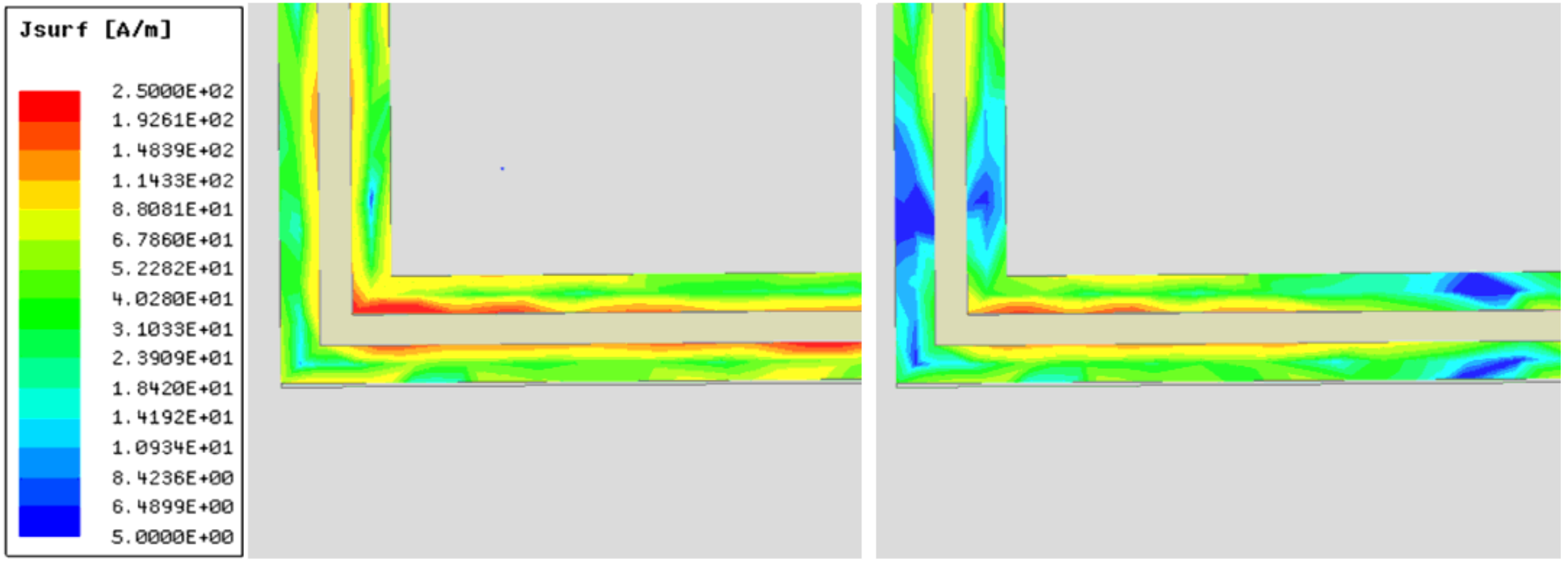

\section{Figure 6}

Surface currents at the dielectric at the frequencies of $2.6 \mathrm{GHz}$ (left) and $8.4 \mathrm{GHz}$ (right). 


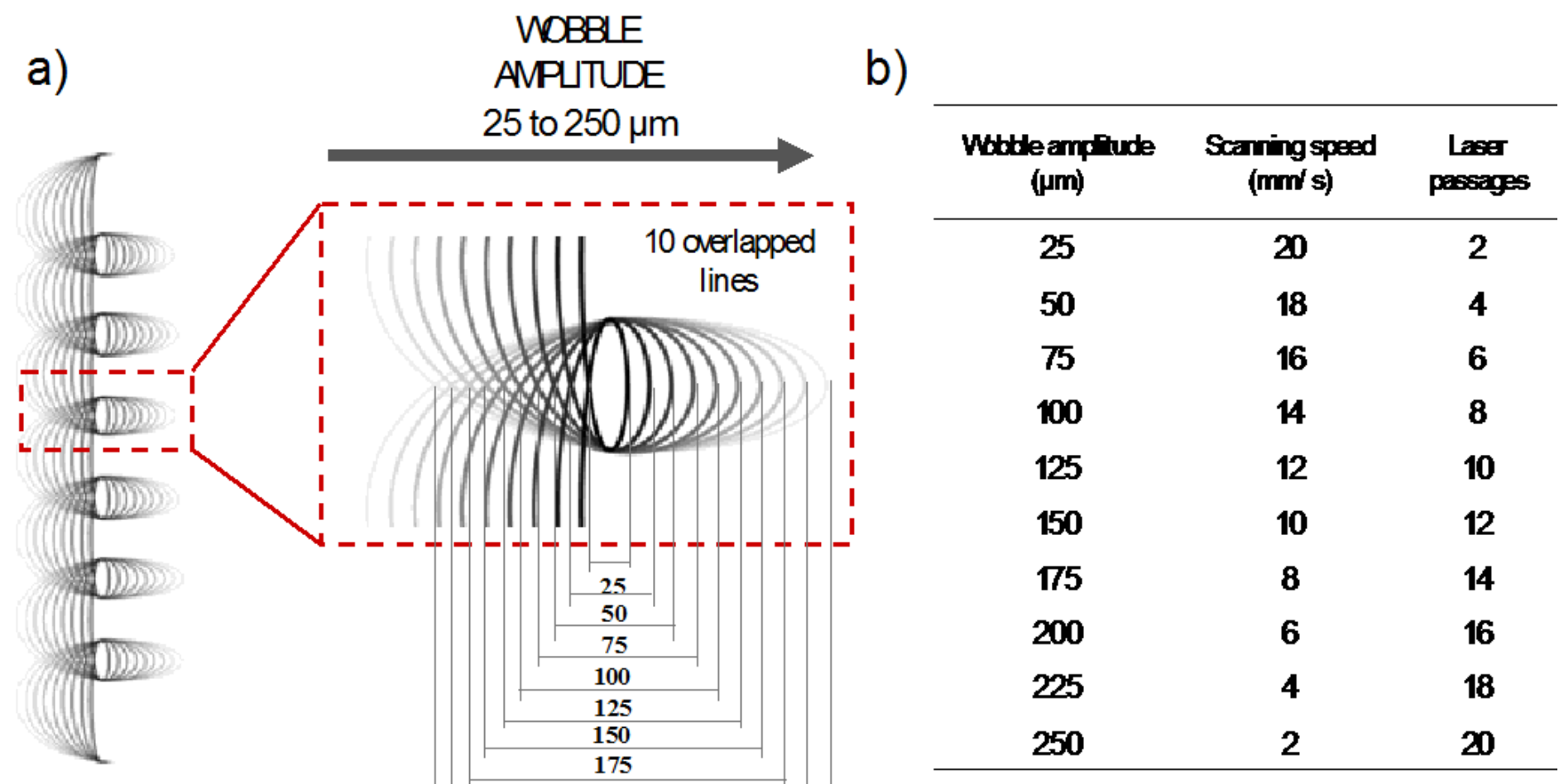

Figure 7

Schematic illustration of the laser strategy used to create the micro-cavity on Ti6Al4V surface. (a) Scheme of the wobbles sequence. (b) Table with the parameters used in each wobble. 
a)

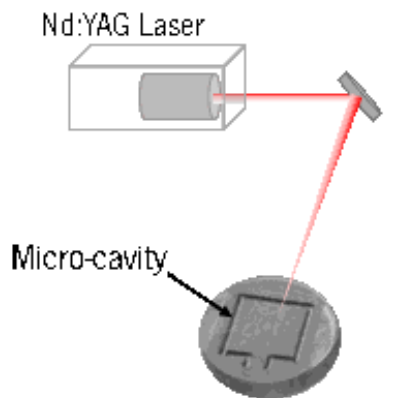

Ti6Al4V substrate b) Step (ii). ZrO2 layer deposition and laser surface heating

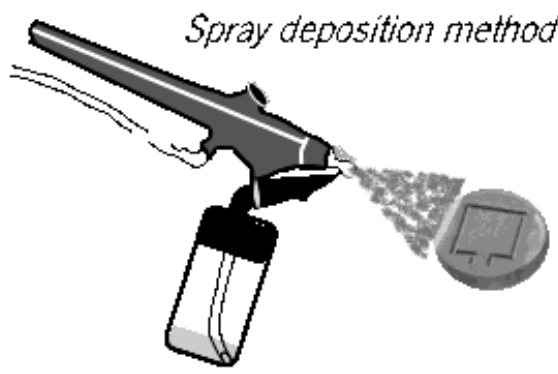

$\mathrm{ZrO}_{2}$ suspension in acetone

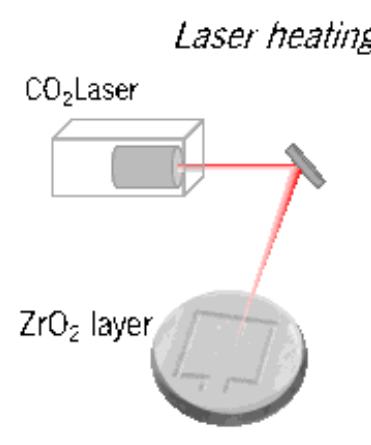

c)

Step (iii). Silver powder deposition and laser sintering

Laser sintering

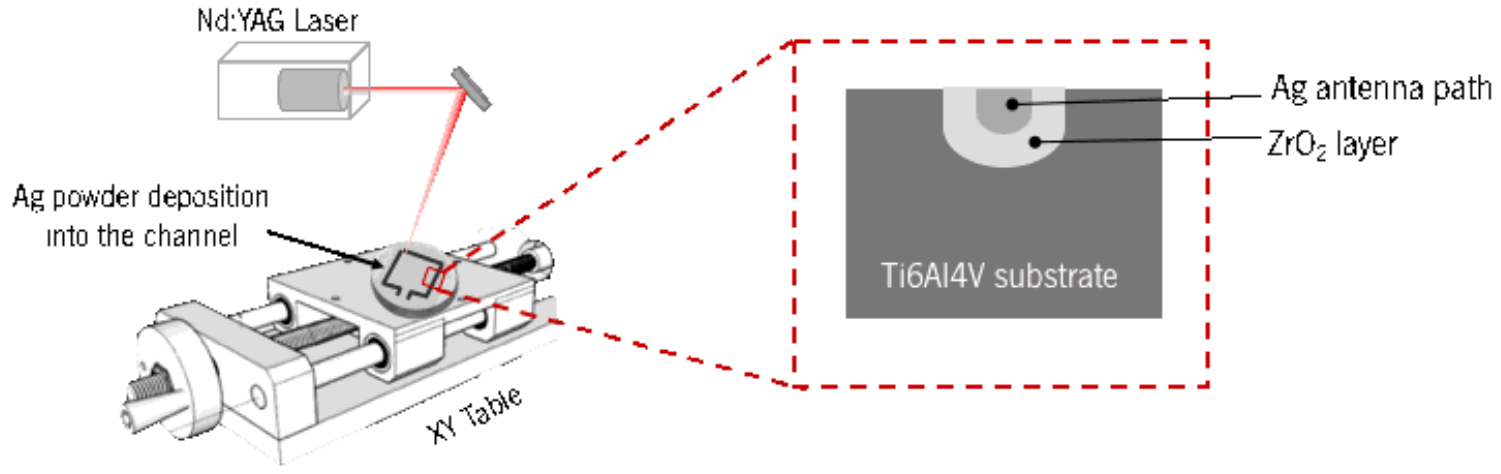

\section{Figure 8}

Schematic illustration of the antenna manufacturing on Ti6Al4V substrate (a-c).

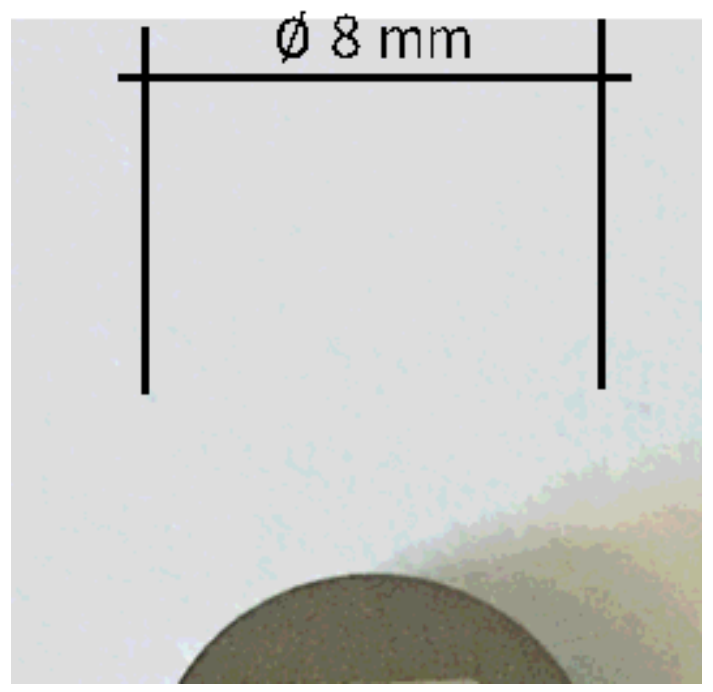

\section{Figure 9}

Photography of the fabricated antenna. 
$250.5 \mu \mathrm{m}$
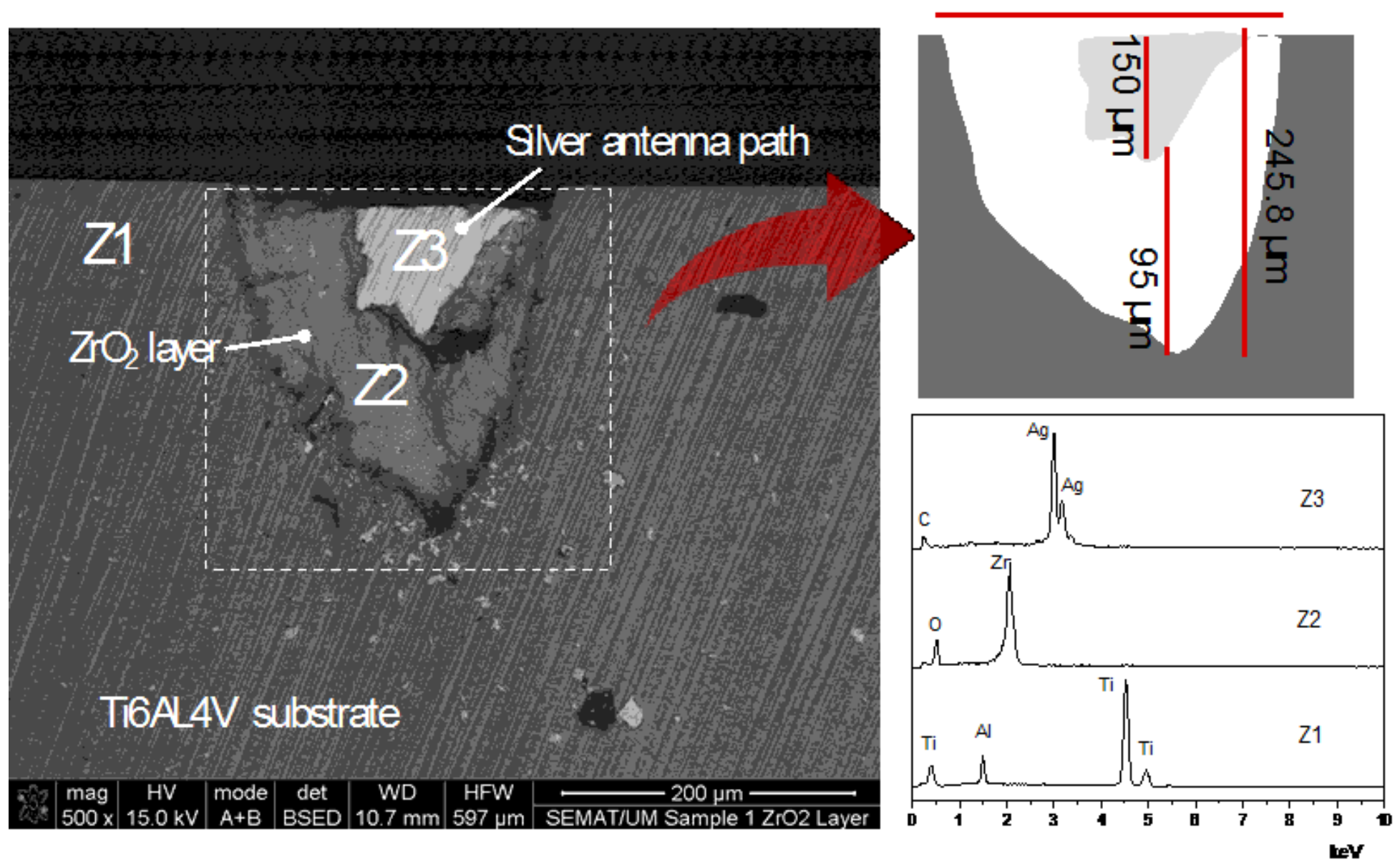

Figure 10

Cross-section SEM images of laser-generated micro-cavity after silver-based antenna manufacturing. On the right side, a schematic illustration of the micro-cavity and the dimensions of the layers, and EDS analysis of each layer.

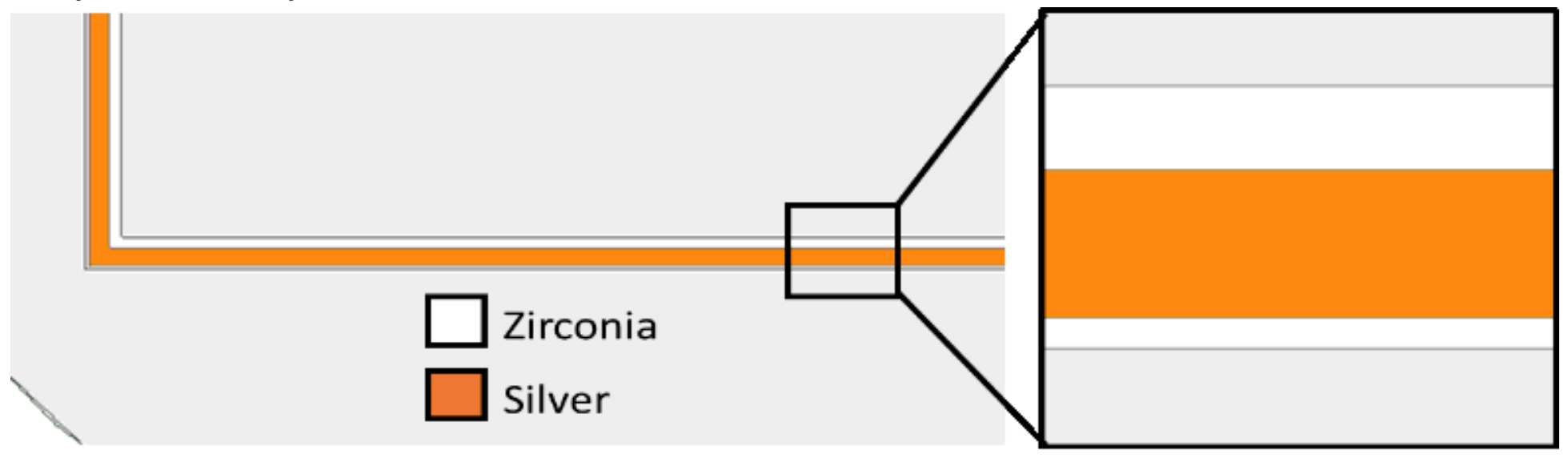

Figure 11

Uncentered silver line detail from the HFSS model of the fabricated antenna. 

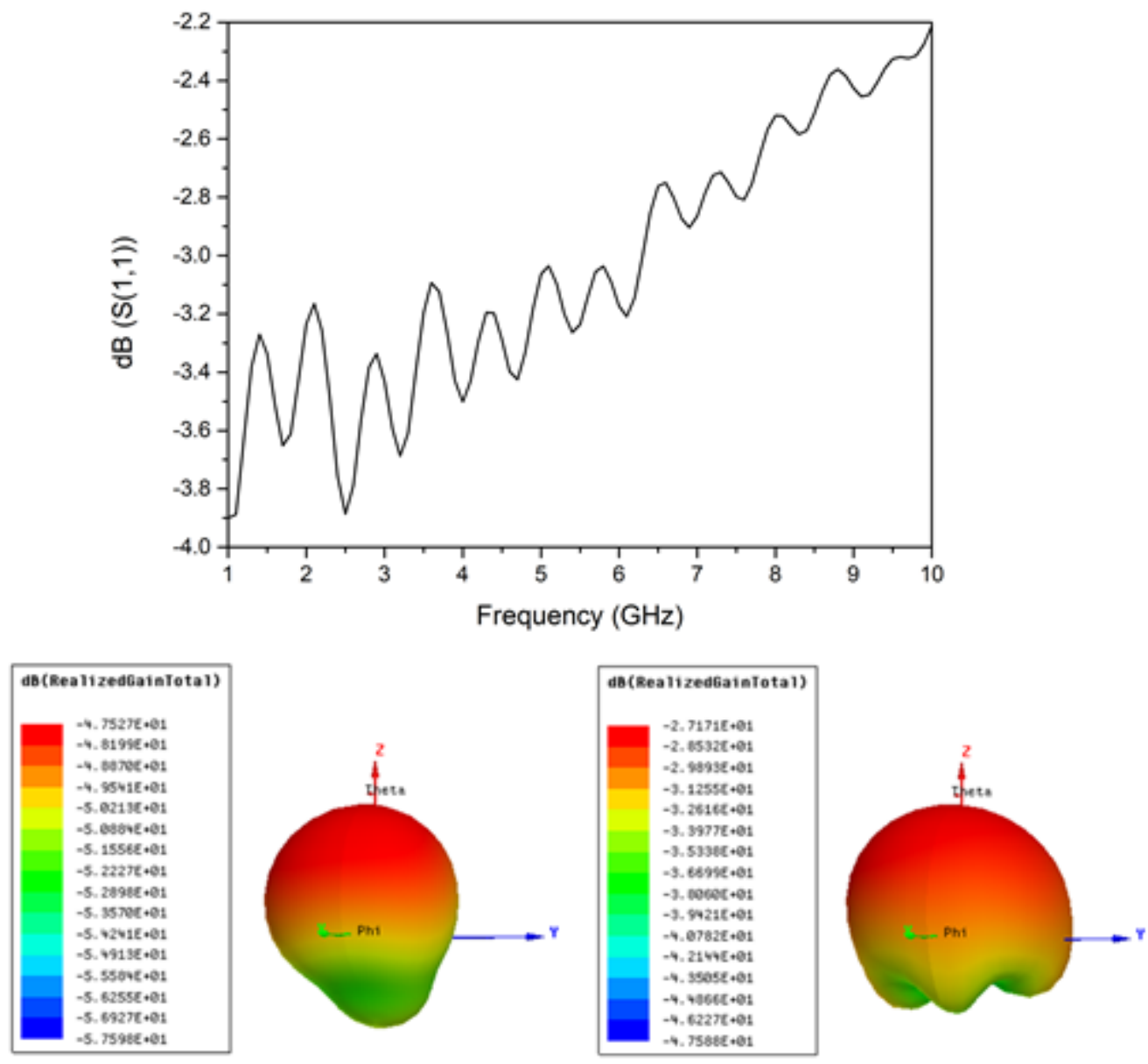

Figure 12

Return loss (top) and radiation patterns of the fabricated antenna at $2.6 \mathrm{GHz}$ (bottom left) and $8.4 \mathrm{GHz}$ (bottom right). 